\title{
Tunable Liquid Crystal-loaded Metasurfaces for IR and THz Applications
}

\author{
O. Buchnev ${ }^{1,3, *}$, J. Wallauer ${ }^{2}$, M. Walther ${ }^{2}$, N. Podoliak ${ }^{1}$, M. Kaczmarek ${ }^{4}$, N. I. Zheludev ${ }^{1,3,5}$ and \\ V. A. Fedotov ${ }^{1,3}$ \\ 1 Optoelectronics Research Centre, University of Southampton, Southampton SO17 1BJ, UK \\ 2 Department of Molecular and Optical Physics, University of Freiburg, D-79104, Germany \\ 3 EPSRC Centre for Photonic Metamaterials, University of Southampton, Southampton SO17 1BJ, UK \\ 4 Physics and Astronomy, University of Southampton, Southampton SO17 1BJ, UK \\ 5 Centre for Disruptive Photonic Technologies, Nanyang Technological University, Singapore 637371, Singapore \\ *e-mail: O.Buchnev@soton.ac.uk
}

\begin{abstract}
We experimentally demonstrate a family of compact and efficient IR and THz electrooptical modulators based on active planar metamaterials (metasurfaces) hybridised with liquid crystals.

OCIS codes: (230.2090) Electro-optical devices; (160.3918) Metamaterials; (160.3710) liquid crystals;
\end{abstract}

\section{Introduction}

Metamaterials in photonics represent a large class of nano-structured artificial media with optical characteristics unavailable, or superior, to those exhibited by natural materials. One of the important steps towards practical applications of the metamaterials is the implementation of an efficient active control over their optical response. It can be achieved by either mechanically changing metamaterial fabric through MEMS or deformations, or hybridizing the metamaterial structure with naturally available nonlinear and functional media. Among the latter the liquid crystals (LCs) have arguably the largest and most broadband optical nonlinearity, whereas their properties can be readily controlled by light, temperature, electric and magnetic fields.

While electrical control of LC properties has been exploited for tuning negative-index microwave metamaterials [1] as well as nonlinear optical metamaterials [2], to the best of our knowledge, we report the first experimental demonstration of efficient low voltage electro-optical modulation and switching in IR and THz planar metamaterials (metasurfaces). This became possible by controlling both micro-scale volume and in-plane ordering of LCs in the resulting hybrid metamaterial systems.

\section{Controlling IR metasurface through volume LC switching}

The regime of micro-scale volume ordering of LCs was achieved by integrating a metasurface into a twisted LC cell. The hybrid structure obtained was composed of a $15 \mu \mathrm{m}$ thick layer of nematic LC E7 confined between the metasurface and a transparent electrode (coated with LC-alignment layer) or another metasurface, as shown in Fig. 1a. The fabric of the metasurface was formed by a continuous zig-zag wire nano-pattern, which was milled using focused ion beam in a $80 \mathrm{~nm}$ thick gold film deposited on a glass substrate (Fig. 1b).

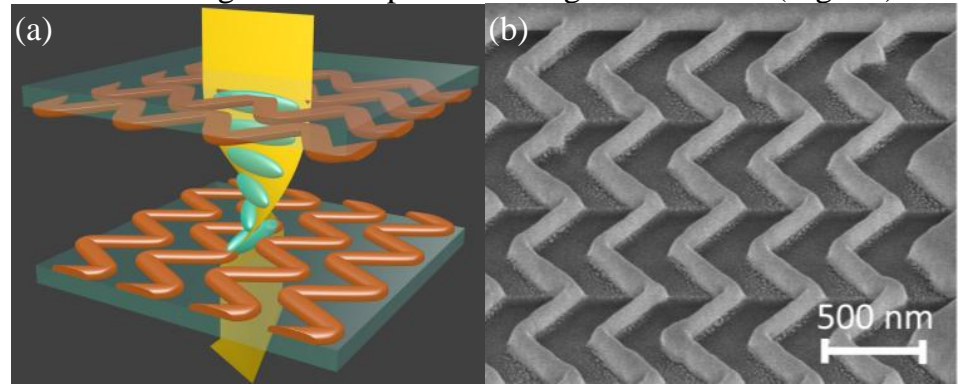

Fig. 1. (a) Artistic view of the hybrid liquid-crystal metamaterial based optical cell, where a zig-zag metamaterial array works as an alignment layer, a polarizer as well as an electrode. (b) SEM micrograph of the metamaterial array taken at $52^{\circ}$ to the array's normal.

Direct contact of liquid crystal with the nano-structure provided anchoring and aligned the LC molecules orthogonally to the molecules at the opposite side of the cell, leading to the volume twisted ordering in the nematic phase. By applying a voltage across the cell of less than $3 \mathrm{~V}$ we were able to reversibly destroy the LC twisted state and reduce transmission of the metamaterial at its resonance by a factor of five. 


\section{Controlling THz metasurface through in-plane LC switching}

Although in-plane switching of an optically thin layer of liquid crystal cannot produce any noticeable transmission effect for THz radiation alone, it can yield a very efficient radiation control mechanism when combined with the strong resonant response of a THz planar metamaterial. We show that an active LC-loaded metasurface exploiting the in-plane LC switching mode enables the control of both intensity and phase of the transmitted terahertz radiation and requires only a moderate driving voltage for its operation.

The metasurface was based on the so-called fish-scale (FS) pattern, a regular array of continuous meandering metallic wires (see Fig. 2a). The fabricated metamaterial array had a square unit cell and a period of $100 \mu \mathrm{m}$, which made it non-diffracting below $1.5 \mathrm{THz}$ for any angle of incidence. To be able to load FS metasurface with LC we placed a quartz cover slide $12 \mu \mathrm{m}$ above the plane of the aluminum pattern, which rendered the resulting structure to be an optically thin cell. The cell was filled with highly birefringent nematic LC 1825 . The surface of the metamaterial and the inner surface of the cover slide were coated with a polymer and rubbed in the direction orthogonal to the straight sections of the meanders, as illustrated in Fig. 2a. The latter promoted uniform alignment of LC molecules in the cell, orthogonal to the incident polarization.
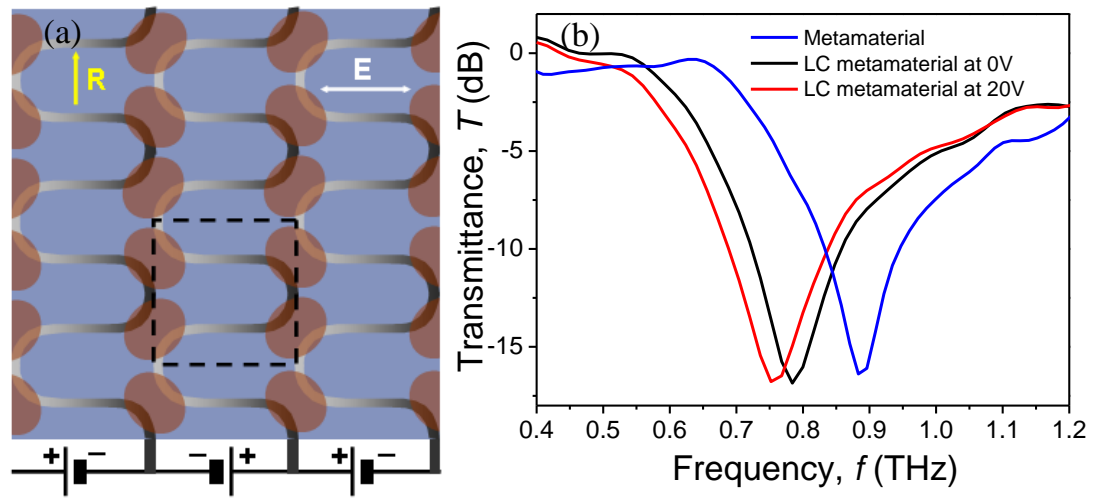

Fig. 2. (a) Schematic of FS planar metamaterial and electric circuitry providing the driving voltage $U_{0}$ for in-plane control of LC. Dashed box indicates the unit cell of the metamaterial array. Shaded areas indicate localizations of in-plane component of applied electrical field.

(b) Transmission spectra both empty and LC-loaded metamaterials.

The transmission response of the metasurface was characterized at normal incidence in the $0.4-1.2 \mathrm{THz}$ range of frequencies using the standard terahertz time-domain spectroscopy technique. The efficient tunability of the metamaterial resonance was achieved in the presence of an in-plane electric field. In particular, by applying a maximum of $20 \mathrm{~V}$ between the meanders we were able to red-shift the resonance frequency by $45 \mathrm{GHz}$. The electrical tuning of the metamaterial resonance resulted in a change of the structure's overall transmission across the entire spectral domain, with a maximum absolute difference in the transmitted intensity $\approx 20 \%$ and the phase $\approx 40$ deg.

\section{Summary}

We experimentally demonstrated efficient electro-optical modulation of the near-IR response of plasmonic metamaterials hybridized with LCs by controlling volume switching of LC-molecules in the resulting hybrid metamaterial system. We also note that the nano-structured metasurface can replace all three essential components of an LC device: (i) LC-alignment layer; (ii) transparent electrode and (iii) polarizer; making the hybrid cell more compact than the conventional LC devices and thus easy to integrate into plasmonic and nano-photonic circuits.

By employing for the first time an in-plane LC switching mode for the THz modulator, we substantially simplified the design of the LC cell. This enabled a reduction of the driving voltage down to a few tens of volts and allowed it to operate in the transmission regime. As the result, we demonstrated the efficient intensity and phase modulation of $\mathrm{THz}$ radiation using an actively controlled metafilm combined with a LC layer only $12 \mu \mathrm{m}$ thick.

\section{References}

[1] Q. Zhao, L. Kang, B. Du, B. Li, J. Zhou, H. Tang, Z. Liang, and B. Zhang, "Electrically tunable negative permeability metamaterials based on nematic liquid crystals," Appl. Phys. Lett. 90, 011112 (2007).

[2] A. Minovich, J. Farnell, D. N. Neshev et al., "Liquid crystal based nonlinear fishnet metamaterials," Appl. Phys. Lett. 100, 121113 (2012). 